\title{
Pulsed Light System for Multiple-Cell Ultracentrifuge Rotor
}

\author{
M. Wales, P. G. Sulzer, and L. C. Williams
}

\begin{abstract}
A system for photographing any spot on a moving rotor is described. A pulsed light, triggered by a signal obtained from a, small alternator on the rotor shaft, is used. By shifting the phase of the light, any desired location on the rotor may be kept in view. This system is used to record data, from an ultracentrifuge having four symmetrically placed cells on the rotor. A scaler-counter circuit is included for speed measurement.
\end{abstract}

\section{Introduction}

In certain types of ultracentrifuge measurements it is desirable to investigate more than one sample at once. Beams ${ }^{1}$ has devised a method for making many sedimentation velocity measurements simultaneously, in order to carry out the Gofman test for lipoprotein on a large number of samples. Sedimentation equilibrium measurements often require a long waiting period for the attainment of equilibrium. ${ }^{2}$ It would, therefore, be advantageous to carry out measurements on as many solutions as possible at the same time, especially when an extrapolation to infinite dilution must be made. ${ }^{3}$ The present paper gives an account of an arrangement that enables four symmetrically placed cells, all containing solutions, to be photographed separately, using the Svedberg ${ }^{4}$ equilibrium machine.

\section{Principle of Operation}

The rotor of late models of this ultracentrifuge has four holes to accommodate three solution cells and a reference cell as shown in figure 1 . The three solution cells are unequally spaced, radially, in order to separate the scale images photographed through each one. This arrangement has two disadvantages: (1) the column of solution is so short that the regions of optical uncertainty near the bottom of the cell and the meniscus comprise a rather large fraction of the cell, and (2) great care must be used in filling the cells when maximum column length is desired, in order to obtain visible menisci. Furthermore, it has been suspected for some time that the use of a separate reference cell, where part of the scale is photographed through air to give reference lines, ${ }^{4}$ is unnecessary. Reference lines photographed through the quartz windows in the upper (unfilled) part of a solution cell gave reproducible reference readings. Therefore, four longer, symmetrically placed solution cells were constructed, ${ }^{5}$ with the cell inserts ${ }^{4}$ of type 316 stainless steel and

$1 \mathrm{~J}$. W. Beams, private communication.

2.M. Wales, J. Applied Phys. 22, 735 (1951).

3 M. Wales, F. T. Adler, and K. E. Van Holde, J. Phys. Colloid Chem. 55. 145 (1951).

${ }^{4} \mathrm{~T}$. Svedberg and K. O. Pedersen, The ultracentrifuge (Oxford Press, Cambridge, England, 1940)

${ }^{3}$ Constructed by R. E. Ward and associates, NBS Shops Division, according to a design originated by $\mathrm{E}$. Hanson, University of W isconsin. the barrels of dural. Dural inserts had given corrosion troubles in aqueous solution and could not be permanently plated. Other types of stainless steel corroded in aqueous salt solutions.

The four cells could be photographed separately by interrupting the light source in such a way as to illuminate the same cell at each flash, while all other cells would be dark. This could be accomplished, in principle, in several ways: (1) mechanically, by gearing a rotating disk with a window in it to the drive-shaft of the motor, and providing suitable means for phase change, (2) electrically, either by driving a rotating disk with a motor synchronized to the centrifuge shaft, or by pulsing a gaseous discharge tube in synchronism. The first method was not tried because of the difficulty of obtaining access to the centrifuge shaft without extensive changes in the design of the machine. However, electrical synchronization appeared promising, because the manufacturer had installed a small coil and magnet alternator on the end of the shaft. This was intended to be used in connection with a pulse-shaping circuit, oscilloscope, and oscillator to measure the speed of rotation. It was found to be simpler and less expensive to feed the output into a scaler circuit with a

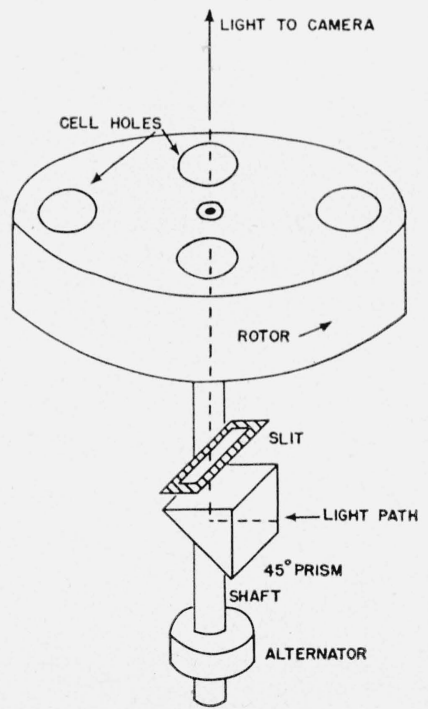

Figure 1. Diagram of ultracentrifuge rotor showing path of light. 


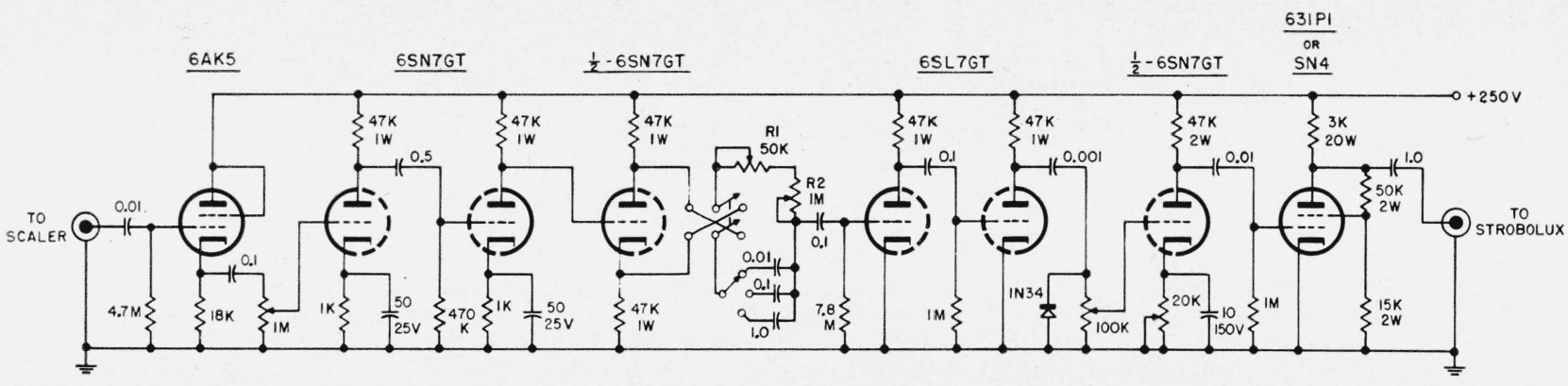

Figure 2. Circuit diagram, coupling of scaler output to Strobolux.

scale of 64 , driving a register, and to count the speed directly. This arrangement was checked for internal consistency by means of an applied 60-cycle pulse (a-c line), the time interval being measured by a synchronous automatic timer, also driven from the line. The circuit was also checked against standard frequencies available at the National Bureau of Standards.

From the 1:8 and 1:16 stages of the scaler, provision was made to tap off the output through a cathode-follower circuit and amplifier, to drive a small synchronous motor. Unfortunately, the wave form obtained was so irregular that the motor would not run in synchronism except at a 1:1 frequency ratio, with the scaler out of the circuit. This made it inadvisable to use the motor because the speed range of the centrifuge is 30 to $300 \mathrm{rps}$, and the motor could be run only at 30 to 80 rps.

The final arrangement, which proved to be successful, is shown in figure 2. A Stroboluxpulsed lightsource assembly was driven with the amplified signal from the centrifuge shaft, via the scaler circuit at a frequency ratio of $1: 8$ (or $1: 16$ if desired). Provision for shifting the phase of the signal by $\pm 180^{\circ}$ was made in order to illuminate any desired cell.
Cells were distinguished by means of identifying dots painted on the far ends of the windows, away from the column of solution. The cell image was centered in the field of view in the camera by means of the potentiometers $R_{1}$ and $R_{2}$, which were brought back to the vicinity of the camera. The images were remarkably stable and remained well centered for as long as $15 \mathrm{~min}$ without adjustment.

Light from the Strobolux was concentrated on the entrance window of the centrifuge optical system by means of a truncated cone of kraft paper covered on the inside with smooth aluminum foil. This material is commonly used for insulation and is readily available. A thin frosted-glass diffuser was necessary for uniform illumination of the scale. A filter, designed originally to isolate the mercury green line, was also used in order to obtain nearly monochromatic light. Under these conditions exposures of 3 to 4 min were required to obtain good scale photographs on CTC Kodaline plates.

It is expected that this arrangement will materially increase the utility of the equilibrium ultracentrifuge.

Washington, December 1, 1952. 